Classification

Physics Abstracts

$07.80-61.46-61.80$

\title{
Letter
}

\section{Shape transformations in single-layer carbon nanotubes}

Pulickel M. Ajayan $\left({ }^{1}\right)$, Christian Colliex $\left({ }^{1}\right)$, Patrick Bernier $\left({ }^{2}\right)$ and Jean-Marie Lambert $\left({ }^{2}\right)$

( $\left.{ }^{1}\right)$ Laboratoire de Physique des Solides, CNRS URA 002, University of Paris-Sud, Bâtiment 510, 91405 Orsay, France

(2) GDPC, CNRS URA 233, Universite Montpellier II Sciences \& Techniques du Languedoc, 34095 Montpellier, France

(Received 21 January 1994; accepted 25 January 1994)

\begin{abstract}
We show that strong electron irradiation of single-layer carbon nanotubes introduces distinct changes in the preferred morphology (shape) of tubes, from perfect cylindrical to a necklace of ridges which result from local deformations of the tube surfaces with the curvature on the order of the tube radii. The shape transformations in these free-standing closed two dimensional surfaces seems to resemble closely the deformations observed in systems such as lipid bilayers and biological membranes.
\end{abstract}

It is now well established that when carbon samples (multi-shell carbon nanotubes [1,2], carbon soot etc.) are subjected to intense electron irradiation, almost spherical graphitic structures result having concentric multi-layers nested one inside the other as if they were onions $[3,4]$. The process results from the concerted migration of the carbon species in the irradiated area through long/short range diffusion inside a carbon matrix towards thermodynamically more stable shapes of graphite particles. Heating of carbon black near melting temperatures or carbonization of some natural polymers under pressure has also been reported [5] to result in spherical or hemi-spherical structures with concentric carbonaceous shells.

However, the monitoring of the exact shape transformations and evolution of the individual layers of the nanotubes to the final onionite stage is an impossible task since inter-layer disordering takes place during irradiation as a precursor stage, forming metastable structures full of defects [6]. Also, the formation of the graphite onions in the final state does not reflect the actual thermodynamic minimum shape that a carbon tube would assume if irradiated or subjected to external stresses but only reflects the minimum shape during the graphitization process of an intermediary disordered structure created out of destroying the original tubular but graphitic lattice of the nanotube. 
In order to study and compare the preferred shape of a tube of carbon (graphite) during externally applied effects such as irradiation one needs to consider the ideal case of a tube that consists of one single layer.

This has become a possibility after single-shell nanotubes having diameters between 1-2 nanometers were successfully grown recently/in the carbon soot, using cobalt and iron catalysts [79]. The structure of these tubes is of a one-dimensional hollow surrounded by a single-layer of graphite skin (or else a two-dimensional closed surface embedded in three dimensional space), with no supporting matrix, and if the carbon atoms present on the surface of such a tube were to move and reorganize, it could do so only along the skin (in two dimensions) unless the tube collapses into a flat piece. There is another possibility that the elastic strains introduced by irradiation (electron irradiation is a complex phenomenon and the effect created by it is almost always non-linear) may transform the tube into unusual shapes. The shape transformation of these free standing single-layer tubes that we report here may be compared to the conformational behaviour of membranes such as lipid vesicles or fluid membranes under strain, a subject of intense interest and reviewed in a recent report [10].

The effect of electron irradiation on single-shell nanotubes was first observed by us in a VG STEM machine (while trying to obtain electron energy loss spectra by focused electron beam) at very high electron fluxes $\left(10^{8} \mathrm{e} / \mathrm{nm}^{2} / \mathrm{s}\right)$. During observation, the tubes were seen "melting" under the beam (it is difficult to distinguish true melting from electron beam induced structural transformations) and breaking along the lengths; the tips of the broken tube segments transformed continuously into spherical (or oblate) shapes, of dimension similar to the tube diameter $(\sim 1.2$ $\mathrm{nm}$ which is the size of majority of the tubes), ejecting these blob-like structures from the tips.

We have reproduced the effect in a high resolution transmission electron microscope (TOPCON 002B), at two orders of magnitude lower fluxes, where the phenomenon can be observed in a more controlled fashion. The observations are shown in figures 1,2 . During irradiation with electrons, the perfectly straight and parallel fringes corresponding to the tube walls become wavy (forming an array of connected ridges) at first, creating necks which grow closer, and then in the final stages produce a necklace of connected beads. It is not clear if complete cross-linking between opposite walls of tubes occurs, which results in the fracture of the tubes or while closed rounded carbon structures detach from the tube tips. The process seems to be similar to the spherodization of very thin (nanometer size) silica fiber tips observed during electron irradiation [11] where spherical ball-like solid structures are formed and ejected from the tips. Here the hollow blobs (if closed) that are formed at the tips should probably correspond to some giant fullerene [12] structures.

Simple heating of the tubes in an inert atmosphere or vacuum do not yield the same results. The tubes remain straight and intact upto $\sim 1600^{\circ} \mathrm{C}$ (when the bulk sample is heated in a furnace) and no morphological changes are observed; the temperature of these thin tubes during electron irradiation should be much smaller than the above value. However, it would be interesting to consider how true melting would proceed and what morphological instability would appear at the onset in such a freely suspended one-dimensional surface structure, if it were heated to the thermodynamic melting point. In both cases (electron beam induced effect and true melting), the morphological instabilities resulting from the introduction of local curvature should be restricted by the possibilities of bending and rotation that the $\mathrm{C}-\mathrm{C}$ bonds can undergo keeping the network continuous. Techniques like scanning tunneling or atomic force microscopy (AFM) should be able to probe such phenomenon in atomic detail and should be able to provide a better understanding of the behaviour of carbon bonds under stress or during phase transition and understand the changes in local structure. A recent AFM study [13] has shown that extremely narrow ridges can be introduced on the surface of nanotubes creating a ripple structure at the atomic level. 

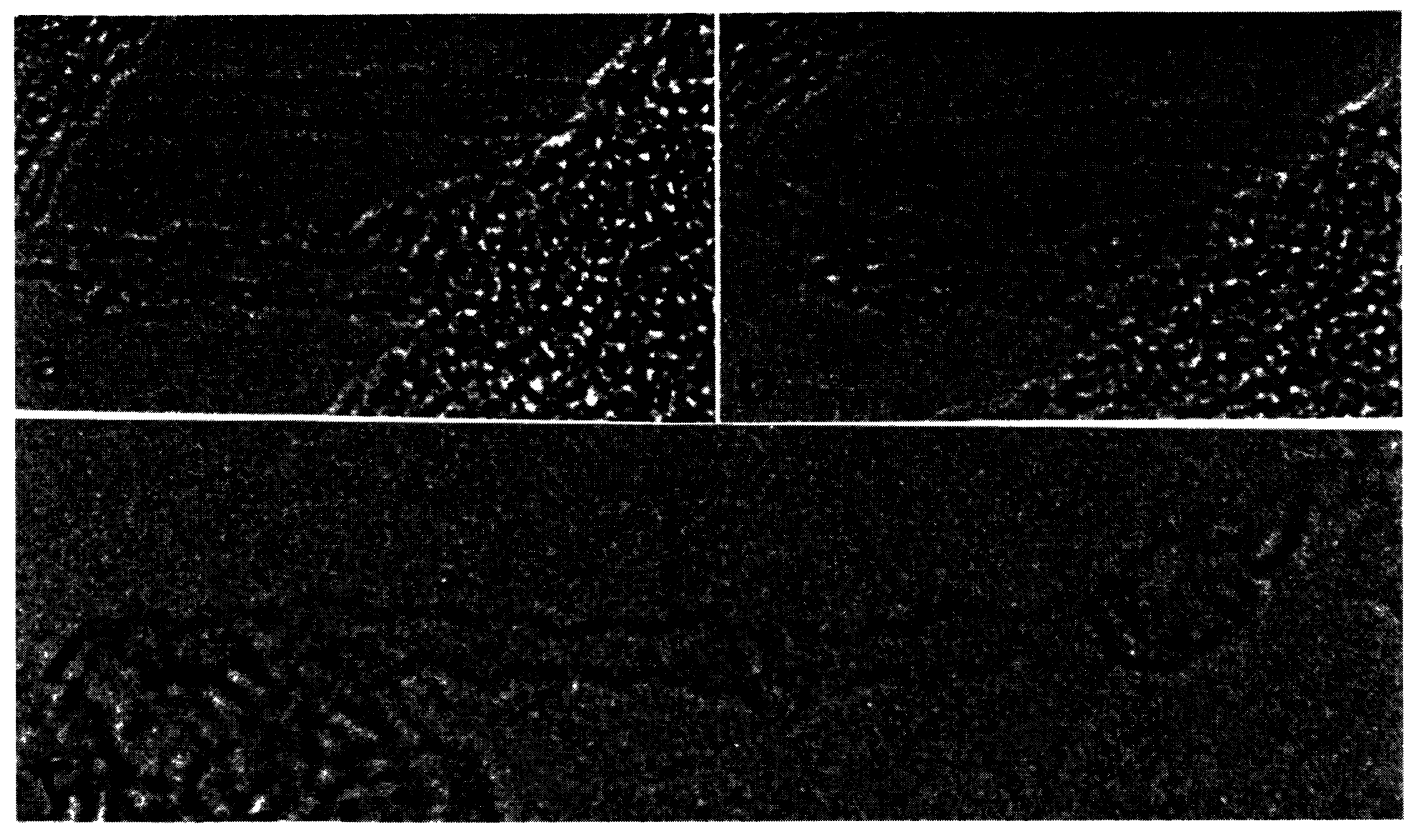

Fig. 1. - Transformation seen in a small segment of single-shell nanotube during electron irradiation. The almost parallel walls of the tube in (a) have become wavy having cusps separated by short necks. (c) a larger tube segment after longer period of irradiation; the tube looks as if it were a sequence of oblate spheroids joined together. The tubes are all $\sim 1.3 \mathrm{~nm}$ in diameter.

Fig. 2. - a) Image of a perfect tube which broke on the left side after irradiation with a high flux electron beam. b) the broken end is seen to undergo a budding transformation and break into smaller fragments which are ejected from the tip. The tube is about $1.5 \mathrm{~nm}$ in diameter. 
This is probably the first time such shape transformations have been directly probed and reported for the microscopic regime in any two dimensional system. In single-layer carbon tubes we have observed that the perfectly straight segments of graphite cylinders (curved in two dimensions) have been transformed into cusps (introduction of local curvature in one extra dimension) of curvature resembling that of the original tube diameter. The necks between these cusps must be interesting areas where minimal surfaces [14] may be present. It is unclear whether the bonds are broken and reformed by the annealing effect under the electron beam. Similar phenomenon of shape instabilities are observed elsewhere in nature in free standing closed membrane systems [10] such as lipid bilayers. In biological systems it is well known that free-standing membranes undergo such budding transitions during conformational instability [10] due to stresses or during phase transitions. Carbon bonds are one of the strongest in nature and yet they seem flexible enough, since in a closed surface such as the nanotube, changes in shapes necessarily mean bond mobility. The role of the electron beam here is perhaps to put excess charges on the surface and create a non-zero surface stress. The tube surface seems to respond to such an effect by changing shape and thereby increasing the total conformational entropy of the system [10].

\section{Acknowledgements.}

One of the authors (PMA) wishes to acknowledge support from the "Grant of French Ministère des Affaires Étrangères". the authors also acknowledge cooperation of the Ile de France TEM network facility.

\section{References}

[1] IIJIMA S., Nature 354 (1991) 56.

[2] EBBESEN T.W. \& AJAYAN P.M., Nature 358 (1992) 220.

[3] UGARTE D., Nature 359 (1992) 707.

[4] KROTO H.W., Nature 359 (1992) 670.

[5] AYACHE J., OBERLIN A. and INAGAKI M., Carbon 28 (1990) 353.

[6] Ugarte D., Chem. Phys. Lett. 207 (1993) 473.

[7] Bethune D.S., KIANG C.H., de VRIES M.S., Gorman G., Savoy R., VazQuez J. and Beyers R., Nature 363 (1993) 605.

[8] IIJIMA S. and ICHIHASHI T., Nature 363 (1993) 603.

[9] Ajayan P.M., Lambert J.M., Bernier P., Barbedette L., Colliex C. and Planeix J.M., Chem. Phys. Lett. 215 (1993) 509.

[10] LIPOWSKI R., Nature 349 (1991) 475.

[11] AJAYAN P.M. and IIJIMA S., Phil. Mag. Lett. 65 (1992) 43.

[12] CURL R.F. and SMALlEY R.E., Sci. Ame. 32 (October 1991).

[13] HiURA H., EBbESEN T.W., FUJITA J., TANIGAKI K. and TAKADA T., Nature 367 (1994) 148.

[14] MACKAY A.L. and TERRONES H., Nature 352 (1991) 762. 